\title{
Acesso e permanência da população LGBT no mercado de trabalho: revisão integrativa
}

\section{Access and permanence of the LGBT population in the labor market: integrative review}

\author{
Alice Melo Silva ${ }^{1 *}$, Almir Gabriel da Silva Fonseca $1^{1}$, Alexia Lins Costa ${ }^{1}$, Bárbara Brasilino \\ de Souza ${ }^{1}$.Jose Wilson Apolinário do Nascimento ${ }^{1}$, Luana Moura Santos ${ }^{1}$, Marcelo Victor Luz \\ Soares ${ }^{1}$ e Ana Larissa Gomes Machado ${ }^{1}$.
}

\begin{abstract}
RESUMO
Este estudo teve como objetivo investigar as barreiras e potencialidades que a população LGBT encontra para o acesso e permanência no mercado de trabalho. Realizou-se uma revisão integrativa da literatura com a seguinte pergunta norteadora: Quais as barreiras e potencialidades de acesso da população LGBT ao mercado de trabalho? Utilizando-se da estratégia PICO. Foram categorizadas como dificuldades para o acesso e permanência das pessoas LGBT no mercado de trabalho: o preconceito vivenciado por pessoas LGBT; e como potencialidades: os avanços das leis anti-discriminatórias, a preocupação quanto à diversificação no mercado de trabalho aliado às novas políticas, e a contribuição de grupos para formar uma sociedade mais justa quanto a reconhecimento e inclusão. Conclui-se que, fatores como o preconceito e até mesmo a marginalização da população LGBT contribuem para dificultar o acesso ao mercado de trabalho. Porém, constata-se que a promoção de um ambiente equitativo e livre destes fatores supracitados, é essencial para a manutenção da inclusão desta população no âmbito do trabalho formal.
\end{abstract}

Palavras-chave: Diversidade; Mercado de trabalho; Organizações.

\section{ABSTRACT \\ ACCESS AND PERMANENCE OF THE LGBT POPULATION IN THE LABOR MARKET: INTEGRATIVE REVIEW}

The objective of this study is to investigate the barriers and potentialities that the LGBT population finds in accessing and remaining in the labor market. An integrative literature review was carried out with the following guiding question: "what are the barriers and potentialities that the LGBT population finds in accessing and remaining in the labor market?" using the PICO strategy. It was categorized as difficulties in accessing and remaining of the LGBT population in the labor market: the discrimination experienced by LGBT people. As for potentialities: the advancement of anti-discrimination laws, the interest in diversity in the labor market along with the new policies, and the contribution of groups to make a fairer society in terms of acceptance and inclusion. In conclusion, factors such as discrimination and even the marginalization of the LGBT population contribute to make it more difficult to enter in the labor market.

\footnotetext{
1 Universidade Federal do Piauí.

*E-mail: alicemellosil@outlook.com;
} 
However, it is verified that promoting an equitable environment, free of the aforementioned factors, is essential to maintain this population inclusion in the scope of formal work.

Keywords: Diversity; Labor market; Organizations.

\section{INTRODUÇÃO}

As organizações estão inseridas em um contexto sistêmico e globalizado, onde as fronteiras geográficas foram rompidas. Nos estudos sobre diversidade sexual e vantagem competitiva realizados por Rohm et al., (2012), evidencia-se o quanto os mercados de trabalho tornaram-se globais e por conseguinte sua competição. Portanto, é cada vez mais necessário investir nas pessoas. Nos diferentes campos de atuação, as singularidades pessoais devem somar, formando um capital intelectual diverso e plural. Entretanto, na prática isso ainda não é uma realidade, especialmente para Lésbicas, Gays, Bissexuais, Travestis e Transsexuais (LGBT) (IRIGARAY; FREITAS, 2011).

É válido ressaltar que, vivências em locais de trabalho plurais e não heterogêneos permitem o compartilhamento de valores e atitudes, favorecem a criatividade dos colaboradores e melhoram o processo decisório da organização. Todavia, tais políticas de diversidade foram compreendidas por mulheres lésbicas como tentativas de assemelhar os empregados, pelo fato de trabalharem na mesma organização; onde, apesar das políticas existirem, não tratam todos com equidade (IRIGARAY; FREITAS, 2011).

O Ministério Público Federal (2017), em sua cartilha sobre igualdade de direitos da população LGBTI, destaca que para as pessoas transexuais e travestis as barreiras são ainda maiores, visto que, além do preconceito e da discriminação existe a marginalização dessa população. Tal afirmativa é reforçada pelo estudo de Nogueira, Aquino e Cabral (2017), o qual evidencia que essa população, em grande parte vive excluída da sociedade, têm suas identidades trans negadas pela imprensa e pelo Estado, não tem acesso a qualificação profissional e não ingressam no mercado de trabalho formal, o que contribui com a segregação desses indivíduos.

De acordo com a Organização Internacional do Trabalho (OIT), o Programa Conjunto das Nações Unidas sobre HIV/Aids (UNAIDS) e o Programa das Nações Unidas para o Desenvolvimento (PNUD), (2015), o direito a trabalhar precisa ser 
garantido a população LGBT; contudo, além de assegurar tal, e a estabilidade no trabalho, é preciso promover um ambiente respeitoso, não discriminatório e equitativo para que todos consigam desenvolver suas competências da melhor maneira.

Mediante o exposto, o estudo teve como objetivo investigar as barreiras e potencialidades que a população LGBT enfrenta para o acesso e a permanência no mercado de trabalho.

\section{METODOLOGIA}

Trata-se de uma revisão integrativa da literatura constituída das seguintes etapas: elaboração do protocolo; avaliação do protocolo; coleta de dados; seleção dos estudos; checagem dos dados coletados; avaliação crítica dos estudos com base no objetivo proposto; síntese dos dados; análise dos dados, resultados e elaboração do artigo; apreciação final, avaliação e sugestões; revisão final a partir de sugestões do orientador.

Mediante a utilização da Estratégia PICO, acrônimo que define para P: população/pacientes; I: intervenção; C: comparação/controle; O: desfecho/outcome, elaborou-se a seguinte pergunta norteadora: Quais as barreiras e potencialidades de acesso da população LGBT ao mercado de trabalho? conforme demonstrado no Quadro 1.

Quadro 1- Elementos da pergunta norteadora com base na estratégia PICo.

\begin{tabular}{|l|l|l|}
\hline Acrônimo & Definição & Descrição \\
\hline P & População de interesse & População LGBT. \\
\hline I & Intervenção & $\begin{array}{l}\text { Oportunidade de acesso e permanência no mercado de trabalho } \\
\text { livre de preconceito e/ou discriminação. }\end{array}$ \\
\hline C & Comparação & $\begin{array}{l}\text { Padrão voltado para a heteronormatividade ou ambiente } \\
\text { equitativo, respeitoso e não discriminatório. }\end{array}$ \\
\hline O & Resultados/desfechos & $\begin{array}{l}\text { As barreiras e potencialidades de acesso e permanência no } \\
\text { mercado de trabalho. }\end{array}$ \\
\hline
\end{tabular}

Fonte: Elaborado pelos autores (2021).

Os critérios de inclusão dos artigos foram: artigos publicados em português, inglês e espanhol, entre os anos de 2010 e 2021, com disponibilidade de texto completos nas 
respectivas bases de dados; sendo excluídos trabalhos acadêmicos ou pesquisas publicadas em anais de congressos ou conferências. Ressalta-se que foi adotada a sigla LGBT para a seleção dos artigos, visto que, a literatura vigente ainda não inclui a sigla LGBTQIA+, utilizada como referência a todas as identidades.

Para a seleção dos artigos foi utilizada a estratégia de busca: "Gender diversity" OR "lgbt population" AND "labor market" AND "organizations" nas seguintes bases de dados: Directory of Open Access Journals (DOAJ), SAGE Journals, Scientific Electronic Library Online (SCIELO) e Academic Search Premier - ASP (EBSCO).

A consulta às bases de dados foi realizada mediante o acesso remoto da Comunidade Acadêmica Federada (CAFe), disponibilizado através do Portal de Periódicos da Coordenação de Aperfeiçoamento de Pessoal de Nível Superior CAPES/MEC, recurso destinado apenas para docentes e graduandos de instituições de ensino superior, que viabiliza o acesso livre a documentos em todas as áreas do conhecimento para fins de pesquisas acadêmicas. As buscas foram realizadas por três pesquisadores de forma independente, a fim de obter maior controle dos resultados encontrados, ao final constatou-se os mesmos resultados.

Após a seleção dos estudos primários, os mesmos foram organizados em listas e exportados de cada base de dados, sendo organizados em pastas. Organizou-se um fluxograma mediante a recomendação Preferred Reporting Items for Systematic Reviews and Meta-Analyses (WARD; USHER-SMITH; GRIFFIN, 2019), a fim de descrever o percurso pelo qual a busca foi executada (figura 1). 
Figura 1 - Fluxograma de seleção dos estudos primários, de acordo com a recomendação PRISMA:

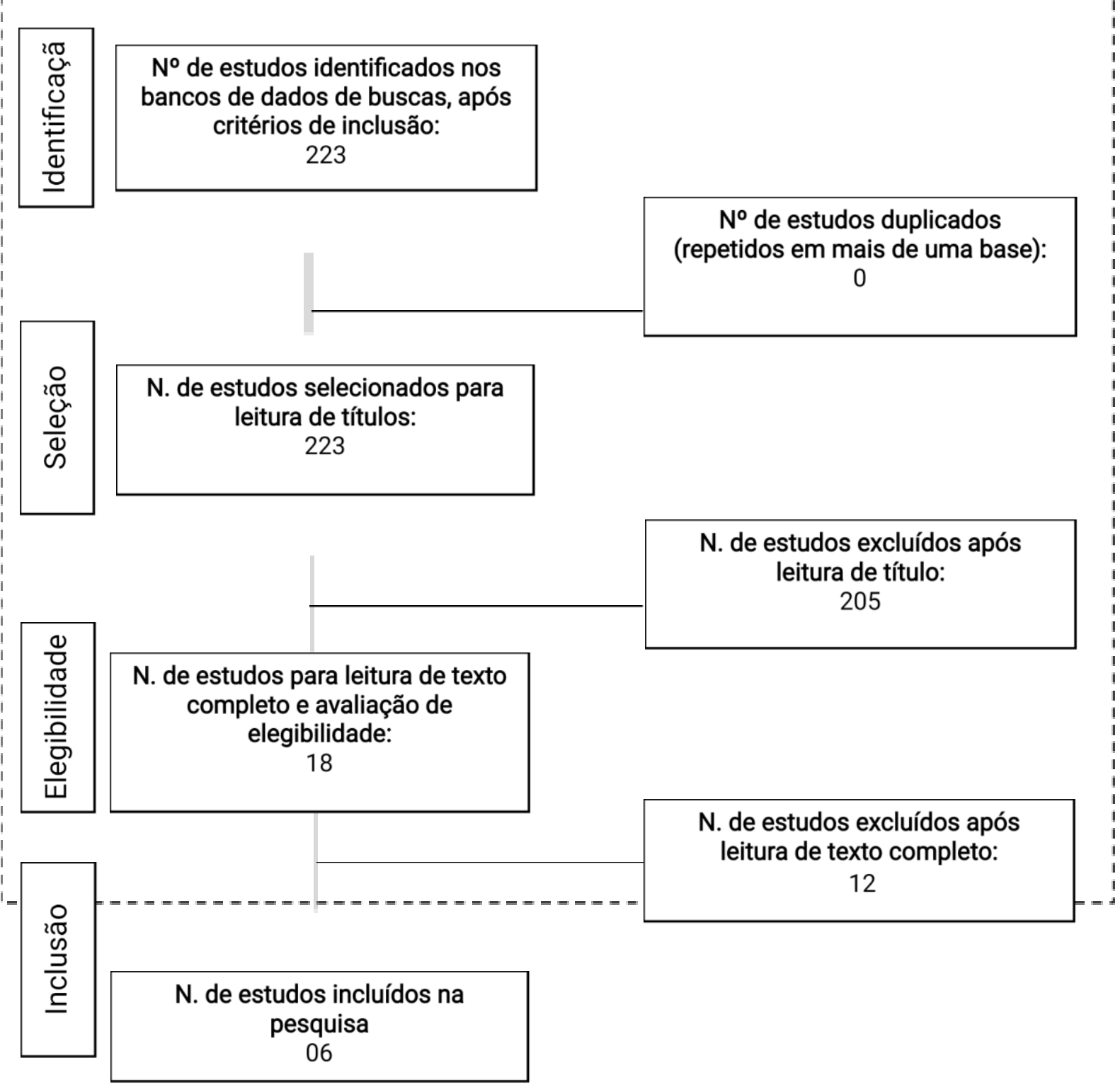

Fonte: Elaborada pelos autores (2021).

Ao todo foram encontrados 223 estudos, ao proceder à leitura dos títulos e resumos e restaram 18 estudos condizentes com a temática, ainda submetidos a leitura por completo para análise de elegibilidade. Após essa fase, um total de 12 artigos foram excluídos por não responderem à pergunta norteadora do estudo. Portanto, foram incluídos na análise 06 estudos para análise distribuídos da seguinte forma em cada base de dados: DOAJ (01), JSTOR (02), SAGE (02) e SCIELO (01).

Desta forma, os artigos foram analisados segundo as variáveis: dificuldades, potencialidades e intervenções propostas. Quanto ao nível de evidência, onde a prática 
baseada em evidências focaliza sistemas de classificação de evidências e geralmente esses sistemas são caracterizados de forma hierárquica, dependendo do delineamento de pesquisa, ou seja, da abordagem metodológica adotada para o desenvolvimento do estudo (WARD; USHER-SMITH; GRIFFIN, 2019). Os artigos foram classificados em: EVANS; GLOVER, (2012) caracterizados como nível de evidência 4 (quatro), que correspondem aos estudos com delineamento não-experimental, como pesquisa descritiva correlacional e qualitativa ou estudos de caso; CORNELIU;, LYNCH, (2014) caracterizado com nível 6 (seis), indicando os estudos baseados em opiniões de autoridades respeitáveis, sendo baseadas na competência clínica ou opinião de comitês de especialistas, incluindo interpretações de informações não respaldadas em pesquisas; KRAUS, P. A.; SCIORTINO, G. (2014), LICCIARDI, N.; WAITMANN, G.; OLIVEIRA, M. H. M. (2015), MIZE, T.D. (2016) e BADGETT, M. V. L.; CARPENTER, C. S.; SANSONE, C. (2021) são caracterizados com o nível de evidência 5, aplicado para relatórios de casos ou dado obtido de forma sistemática, de qualidade verificável ou dados de avaliação de programas (STILLWELL; FINEOUT-OVERHOLT; MELNYK; WILLIAMSON, 2010).

\section{RESULTADOS E DISCUSSÃO}

Buscando sintetizar os resultados encontrados nos estudos selecionados, foi elaborado o quadro 2 que apresenta a caracterização conforme seus códigos, ano e local de publicação, metodologia, público-alvo, nível de evidência e objetivos dos trabalhos.

Quadro 2- Caracterização dos estudos analisados:

\begin{tabular}{|l|l|l|l|l|}
\hline Código & Ano/País & Método/participantes & NE & Objetivos \\
\hline A1 & 2012, Chile & $\begin{array}{l}\text { Revisão narrativa/ } \\
\text { Organizações parceiras. } \\
\text { J., 2012. }\end{array}$ & 4 & $\begin{array}{l}\text { Promover um debate crítico } \\
\text { acerca da eficácia de uma } \\
\text { abordagem de gestão da } \\
\text { diversidade para promover } \\
\text { mudança significativa nesta } \\
\text { área. }\end{array}$ \\
\hline
\end{tabular}




\begin{tabular}{|c|c|c|c|c|}
\hline $\begin{array}{l}\text { A2 } \\
\text { CORNELIUS, J. V.; } \\
\text { LYNCH, I., } 2014 .\end{array}$ & $\begin{array}{ll}2014, \quad \text { África } \\
\text { do Sul }\end{array}$ & $\begin{array}{l}\text { Revisão narrativa com } \\
\text { declaração de posição da } \\
\text { diversidade sexual e de } \\
\text { gênero PsySSA / População } \\
\text { LGBTI da África do Sul e } \\
\text { profissionais da área da } \\
\text { Psicologia. }\end{array}$ & 6 & $\begin{array}{l}\text { Compreender os desafios } \\
\text { que os indivíduos LGBTI } \\
\text { enfrentam em sociedades } \\
\text { patriarcais } \\
\text { heteronormativas que } \\
\text { discriminam com base na } \\
\text { sexualidade e no gênero. }\end{array}$ \\
\hline $\begin{array}{l}\text { A3 } \\
\text { KRAUS, P. A.; } \\
\text { SCIORTINO, G., } 2014 .\end{array}$ & 2014, Itália & $\begin{array}{l}\text { Reflexão teórica narrativa } \\
\text { com instituições da União } \\
\text { Europeia. }\end{array}$ & 5 & $\begin{array}{l}\text { Analisar como as políticas } \\
\text { europeias têm lidado com as } \\
\text { quatro dimensões-chave da } \\
\text { diversidade social: língua, } \\
\text { religião, imigração e } \\
\text { sexualidade de gênero. }\end{array}$ \\
\hline $\begin{array}{l}\text { A4 } \\
\text { LICCIARDI, } \\
\text { WAITMANN, } \\
\text { OLIVEIRA, M. H. M.; } \\
2015 .\end{array}$ & 2015, Brasil & $\begin{array}{l}\text { Pesquisa qualitativa e } \\
\text { investigação exploratória / } \\
\text { Mulheres travestis e } \\
\text { transexuais brasileiras }\end{array}$ & 5 & $\begin{array}{l}\text { Expor a empregabilidade } \\
\text { atual das travestis e } \\
\text { transexuais no Brasil e } \\
\text { propor uma nova } \\
\text { perspectiva para que as } \\
\text { organizações se tornem } \\
\text { ambientes inclusivos e } \\
\text { diversificados, combatendo } \\
\text { o preconceito e a } \\
\text { discriminação que esse } \\
\text { grupo enfrenta, priorizando } \\
\text { o debate e as investigações } \\
\text { em torno da mulher travesti } \\
\text { e transexual, abordando a } \\
\text { sua discriminação no } \\
\text { mercado de trabalho. }\end{array}$ \\
\hline
\end{tabular}




\begin{tabular}{|l|l|l|l|l|}
\hline A5 & $\begin{array}{l}\text { 2016, Estados } \\
\text { MIZE, T.D., 2016. }\end{array}$ & $\begin{array}{l}\text { Estudo qualitativo } \\
\text { População LGBT }\end{array}$ & 5 & $\begin{array}{l}\text { Analisar a proporção de } \\
\text { ganhos salariais da } \\
\text { população LGBT em } \\
\text { relação aos ganhos da } \\
\text { população heterossexual no } \\
\text { mercado de trabalho Norte } \\
\text { Americano. }\end{array}$ \\
\hline $\begin{array}{l}\text { A6 } \\
\text { BADGETT, M. V. L.; } \\
\text { SANSONE, C., 2021. }\end{array}$ & Unidos & $\begin{array}{l}\text { LGBTQ } \\
\text { Revisão narrativa / Pessoas }\end{array}$ & 5 & $\begin{array}{l}\text { Examinar os dados de } \\
\text { pesquisas recentes para } \\
\text { quantificar o número de } \\
\text { pessoas LGBTQ discutir a } \\
\text { qualidade desses dados, nos } \\
\text { Estados Unidos e em } \\
\text { comparação com indivíduos } \\
\text { heterossexuais cisgêneros. }\end{array}$ \\
\hline
\end{tabular}

Fonte: Elaborado pelos autores (2021).

O quadro apresenta estudos publicados de 2012 a 2021, distribuídos em países como África do Sul, Brasil, Chile, Estados Unidos e Itália. Caracterizam-se como estudos de revisão narrativa da literatura e com abordagem qualitativa. Percebe-se que quatro dos seis estudos têm como público alvo a população LGBT, com enfoque em pessoas transexuais associando à suas dificuldades do mercado de trabalho, os outros dois estudos abordam a diversidade social. Quanto aos objetivos, é analisada a objeção que a comunidade LGBT enfrenta desde a criminalização da classe até as dificuldades e desafios encontrados no meio trabalhista, além de explanar sobre a importância da diversidade e inclusão.

A partir disso, foram categorizadas as dificuldades encontradas nas pesquisas e estudos analisados, como também as potencialidades, e propostas para solucionar a problemática apresentadas no quadro 3. 
Quadro 3 - Dificuldades, potencialidades e proposições sobre a inclusão da população LGBT no mercado de trabalho:

\begin{tabular}{|c|c|c|c|}
\hline Código & Dificuldades & Potencialidades & Proposições \\
\hline A1 & $\begin{array}{l}\text { Dificuldade de implementação } \\
\text { da prática nas empresas que } \\
\text { podem escolher defender } \\
\text { iniciativas em razão de suas } \\
\text { necessidades individuais, por } \\
\text { comodismo e anulando seu real } \\
\text { sentido. }\end{array}$ & $\begin{array}{l}\text { A gestão da diversidade pode } \\
\text { ser considerada como uma } \\
\text { resposta racional à condução } \\
\text { de negócios em um mercado de } \\
\text { trabalho global competitivo. }\end{array}$ & $\begin{array}{l}\text { Necessidade de outros tipos } \\
\text { de gestão, sugerindo que } \\
\text { sigam um modelo mais } \\
\text { alternativo e flexível, } \\
\text { levando a uma atuação } \\
\text { sistêmica capaz de solucionar } \\
\text { os problemas mais } \\
\text { complexos e promover a } \\
\text { inclusão das pessoas LGBT. }\end{array}$ \\
\hline A2 & $\begin{array}{l}\text { Em muitos países africanos há } \\
\text { discriminação ativa, e até } \\
\text { mesmo processo e perseguição } \\
\text { de lésbicas, gays, bissexuais, } \\
\text { transgêneros e intersexuais } \\
\text { (LGBTI) são a norma. }\end{array}$ & $\begin{array}{l}\text { Mediante a adoção de uma } \\
\text { postura afirmativa em relação à } \\
\text { diversidade sexual e de gênero, } \\
\text { os profissionais de psicologia } \\
\text { podem desempenhar um papel } \\
\text { fundamental na transformação } \\
\text { de sistemas sexuais e de gênero } \\
\text { injustos, e os efeitos } \\
\text { prejudiciais que estendem-se } \\
\text { além de sua influência sobre } \\
\text { indivíduos LGBTI, queer e } \\
\text { assexuais, para todas as } \\
\text { pessoas. }\end{array}$ & $\begin{array}{l}\text { Respeitar os direitos } \\
\text { humanos das pessoas quanto } \\
\text { às questões de gênero e } \\
\text { sexualidade, e } \\
\text { comprometer com a não } \\
\text { discriminação com base } \\
\text { nessas características. } \\
\text { Essas diretrizes de prática } \\
\text { sul-africanas } \\
\text { profissionais de tecnologia } \\
\text { serão acompanhadas } \\
\text { utilizadas na África para o } \\
\text { desenvolvimento } \\
\text { declarações e diretrizes } \\
\text { práticas adequadas aos seus } \\
\text { contextos únicos. }\end{array}$ \\
\hline A3 & $\begin{array}{l}\text { Nos países da União Europeia } \\
\text { a distribuição desigual de } \\
\text { recursos e direitos entre as } \\
\text { categorias sociais podem ser } \\
\text { limitantes para a eco- } \\
\text { competitividade económica e } \\
\text { geopolítica na cena global. }\end{array}$ & $\begin{array}{l}\text { A referência crescente à } \\
\text { diversidade como um quadro } \\
\text { conceitual } \\
\text { particularmente } \\
\text { correspondido } \\
\text { fortalecimento de redes de } \\
\text { relacionamentos duradouros } \\
\text { entre as Instituições europeias } \\
\text { e autoridades locais, têm }\end{array}$ & $\begin{array}{l}\text { Reconhecimento da } \\
\text { diversidade histórica e } \\
\text { cultural dos Estados } \\
\text { membros; como proteção do } \\
\text { indivíduo e diversidade de } \\
\text { grupo dentro de uma esfera } \\
\text { pública europeia } \\
\text { supostamente compartilhada } \\
\text { e igualitária; como um }\end{array}$ \\
\hline
\end{tabular}




\begin{tabular}{|c|c|c|c|}
\hline & & $\begin{array}{l}\text { contribuído para alargar o } \\
\text { âmbito do significado da } \\
\text { diversidade. }\end{array}$ & $\begin{array}{l}\text { instrumento para alcançar a } \\
\text { estabilização política, a } \\
\text { competitividade do mercado, } \\
\text { e crescimento econômico. }\end{array}$ \\
\hline A4 & $\begin{array}{l}\text { Travestis e transexuais são } \\
\text { excluídas do mercado de } \\
\text { trabalho, como se não fossem } \\
\text { cidadãs capazes e talentosas, } \\
\text { interessadas em carreira, } \\
\text { realização profissional e } \\
\text { ascensão pessoal e, como se } \\
\text { elas não fossem importantes } \\
\text { para a construção de um } \\
\text { ambiente diversificado e livre } \\
\text { de preconceitos. }\end{array}$ & $\begin{array}{l}\text { No cenário organizacional } \\
\text { atual, há uma grande } \\
\text { preocupação por parte das } \\
\text { organizações em reter talentos } \\
\text { e diversificar o ambiente de } \\
\text { trabalho, de modo a aumentar } \\
\text { seu capital intelectual e, } \\
\text { consequentemente, o seu valor } \\
\text { no mercado. }\end{array}$ & $\begin{array}{l}\text { Isolando apenas o ambiente } \\
\text { laboral, faz-se necessário a } \\
\text { implementação de políticas e } \\
\text { práticas de recursos humanos } \\
\text { para que o ambiente de } \\
\text { trabalho torne-se mais aberto } \\
\text { e inclusivo para esses } \\
\text { profissionais. A necessidade } \\
\text { de estruturar o ambiente de } \\
\text { trabalho desde os aspectos } \\
\text { mais básicos, como o uso do } \\
\text { nome social, o uso do } \\
\text { banheiro de acordo com a } \\
\text { identidade de gênero, o } \\
\text { treinamento } \\
\text { colaboradores dos } \\
\text { desconstruir quaisquer tipos } \\
\text { de preconceitos e até políticas } \\
\text { de gestão da diversidade } \\
\text { devem ser implementados } \\
\text { pelos recursos humanos } \\
\text { estratégicos, visando agregar } \\
\text { valor à companhia. }\end{array}$ \\
\hline A5 & $\begin{array}{l}\text { Homens gays e indivíduos } \\
\text { bissexuais } \\
\text { desvantagens no mercado de } \\
\text { trabalho devido ao tratamento } \\
\text { desigual e à estigmatização, e } \\
\text { ao acesso diferenciado às } \\
\text { empresas. As minorias sexuais } \\
\text { enfrentam uma série de } \\
\text { desvantagens e visões } \\
\text { prejudiciais, fatores que } \\
\text { provavelmente contribuem }\end{array}$ & $\begin{array}{l}\text { Dentre a população LGBT, } \\
\text { neste estudo, as mulheres } \\
\text { lésbicas constituem uma breve } \\
\text { exceção, pois constatou-se que } \\
\text { desempenham um bom } \\
\text { resultado na comparação } \\
\text { salarial com mulheres } \\
\text { heterossexuais, apresentando } \\
\text { vantagens pontuais na análise } \\
\text { em questão. }\end{array}$ & $\begin{array}{l}\text { Avanços na formulação de } \\
\text { políticas públicas que } \\
\text { busquem promover equidade } \\
\text { de direitos e benefícios, uma } \\
\text { vez que a discriminação no } \\
\text { mercado de trabalho com } \\
\text { base no sexo e orientação } \\
\text { permanece não-legal na } \\
\text { maioria dos estados nos } \\
\text { Estados Unidos. }\end{array}$ \\
\hline
\end{tabular}




\begin{tabular}{|c|c|c|c|}
\hline & $\begin{array}{l}\text { para um mercado de trabalho } \\
\text { desigual, como as diferenças } \\
\text { salariais observadas. }\end{array}$ & & \\
\hline A6 & $\begin{array}{l}\text { Os estudos mostraram que } \\
\text { essas pessoas têm uma } \\
\text { probabilidade menor de serem } \\
\text { selecionadas para uma } \\
\text { entrevista de trabalho. Em } \\
\text { relação a questão salarial os } \\
\text { estudos evidenciaram as } \\
\text { diferenças salariais negativas } \\
\text { entre homens cisgenero } \\
\text { heterossexuais e homens gays e } \\
\text { bissexuais, já a comparação } \\
\text { entre mulheres heterossexuais } \\
\text { e lésbicas ou bissexuais trouxe } \\
\text { diferenças positivas para } \\
\text { mulheres dessa segunda } \\
\text { categoria. Além disso, os } \\
\text { estudos com pessoas } \\
\text { transgênero ainda são restritos. }\end{array}$ & $\begin{array}{l}\text { Avanços com as leis anti } \\
\text { discriminatórias adotadas por } \\
\text { alguns países, } \\
\text { reconhecimento legal do } \\
\text { casamento entre pessoas do } \\
\text { mesmo sexo. }\end{array}$ & $\begin{array}{l}\text { Inclusão de questionamentos } \\
\text { acerca do gênero e da } \\
\text { sexualidade em censos } \\
\text { demográficos, bem como o } \\
\text { estudo desses fatores em } \\
\text { países em desenvolvimento, } \\
\text { para entender essas } \\
\text { variações. }\end{array}$ \\
\hline
\end{tabular}

Fonte: Elaborado pelos autores (2021).

Portanto, essas foram as dificuldades, potencialidades e intervenções propostas nos artigos selecionados para análise. E assim, adiante, cada um destes pontos supracitados são discutidos a fim de abordar e compreender melhor suas especificidades. As dificuldades encontradas relacionam-se ao preconceito vivenciado por pessoas LGBT, pois esses fatores são acompanhados por indiferenças, perseguições e desvantagens. No âmbito judicial, já existem leis asseguradas que punem e proíbem os atos de preconceito e discriminação. No Brasil, a Lei no 7.716, de 5 de janeiro de 1989, estabelece que "serão punidos, na forma desta Lei, os crimes resultantes de discriminação ou preconceito de raça, cor, etnia, religião ou procedência nacional”. Ainda, a Constituição da República Federativa do Brasil de 1988 determina que:

Constituem objetivos fundamentais da República Federativa do Brasil: I-construir uma sociedade livre, justa e solidária; II-garantir o desenvolvimento nacional; IIIerradicar a pobreza e a marginalização e reduzir as desigualdades sociais e regionais; IV- 
promover o bem de todos, sem preconceitos de origem, raça, sexo, cor, idade e quaisquer outras formas de discriminação. (Art. 3º, I, II, III e IV, 1988).

Mesmo assim, em pleno século XXI, constatam-se inúmeros casos desses tipos de violência. Em associação, às pessoas LGBT são discriminadas, até mesmo marginalizadas pela sociedade e, em muitos casos, pela própria família, criando assim inúmeras dificuldades para receberem apoio psicológico, educação e posteriormente, quando adultas ingressarem no mercado formal de trabalho (BENTO, 2008).

Essa comunidade enfrenta diariamente dificuldades no meio social e laboral, não apenas quanto à violação de seus direitos, mas também quanto ao respeito e inclusão. Neste caso, destacando apenas o âmbito do mercado de trabalho formal, podemos notar que o contingente de mulheres travestis e transexuais em qualquer área de atuação são extremamente inferiores ao de homens e mulheres cisgêneros, inclusive homossexuais (LICCIARD; WAITMANN; OLIVEIRA, 2015).

Vale ressaltar que as pessoas transexuais têm dificuldade também quanto a sua identificação, pois o mercado de trabalho é um contexto que desfavorece e exclui esses indivíduos, alimentando a marginalização e preconceito. De acordo com Foucault (1988), são disseminados diferentes mecanismos de controle dentro sociedade e instrumentos acabam por impor uma identidade aos sujeitos, mesmo contra sua vontade. Esses dispositivos são responsáveis por manter certo domínio sobre a vida dos indivíduos, de modo que é concebida uma divisão do mundo entre heterossexuais e homossexuais; sujeitos morais e imorais.

As potencialidades perceptíveis nos artigos analisados estão associadas aos avanços das leis anti-discriminatórias, a preocupação quanto à diversificação no mercado de trabalho aliado às novas políticas, e a contribuição de grupos para formar uma sociedade mais justa quanto a reconhecimento e inclusão. Portanto, as organizações que procuram uma adoção da diversidade em seu âmbito investem em novos programas de recrutamento e seleção, bem como novas estratégias de comunicação e inclusão e foco em treinamento, visto que uma gestão da diversidade proporciona um capital de reputação positivo, além de alcançar três importantes pilares organizacionais, como: responsabilidade social, melhoria dos resultados e obrigações legais (SALES, 2017).

Quanto às intervenções propostas, destaca-se o respeito e reconhecimento da diversidade, efetuar políticas públicas de recursos humanos bem como adequar o ambiente de trabalho para receber pessoas LGBTQI e treinamentos para desconstruir o 
preconceito; uma vez que, a melhora dos resultados é defendida a partir da razão de que ambientes diversos são caracterizados por indivíduos criativos, com boa comunicação e uma harmônica relação de trabalho (SOUZA, 2021). Ademais, recomenda-se a inclusão de questionamentos quanto ao gênero e sexualidade em censos demográficos, para entender o desenvolvimento desse grupo nos países que ainda não adotaram tal medida.

\section{CONSIDERAÇÕES FINAIS}

Esta revisão mostrou que a comunidade LGBT enfrenta dificuldades para o ingresso no âmbito formal de trabalho. Os estudos analisados confirmam que esse fato não é restrito ao Brasil e com isso trazem a emergência de políticas organizacionais que proporcionem não somente a entrada, mas a permanência e o desenvolvimento de soft $e$ hard skills no ambiente de trabalho. A diversidade se torna o caminho para uma sociedade mais justa, bem como para o desenvolvimento da potencialidade humana no ambiente de trabalho.

Evidenciou-se que fatores como o preconceito e até mesmo a marginalização da população LGBT contribuem para dificultar o acesso ao mercado de trabalho. Constatase que a promoção de um ambiente equitativo e livre destes fatores é essencial para a manutenção da inclusão desta população no âmbito do trabalho formal.

Ressalta-se que o tema carece de estudos com uma maior série temporal e uma investigação em campo, com destaque à população trans, pois ainda é invisibilizada. Em suma, o presente estudo contribuiu para a síntese do conhecimento acerca da temática e sugere a ampliação das pesquisas de intervenção na área das ciências sociais aplicadas para favorecer o acesso equitativo da população LGBT ao mercado laboral.

\section{REFERÊNCIAS}

BRASIL. Constituição (1988). Constituição da República Federativa do Brasil. Brasília, DF: Senado Federal: Centro Gráfico, 1988.

BRASIL. O Ministério Público e a Igualdade de Direitos para LGBTI: Conceitos e Legislação / Procuradoria Federal dos Direitos do Cidadão, Ministério Público do Estado do Ceará. - 2. ed., rev. e atual. - Brasília: MPF, 2017.

BENTO, B. O que é transexualidade. São Paulo: Brasiliense, 2008.

FOUCAULT, Michel. História da Sexualidade I: A Vontade de Saber. Rio de Janeiro, Edições Graal, 1988. 
IRIGARAY, Hélio Arthur Reis; FREITAS, Maria Ester de. Sexualidade e organizações: estudo sobre lésbicas no ambiente de trabalho. Organizações \& Sociedade, v. 18, n. 59, p. 625-641, 2011.

LICCIARDI, Norma; WAITMANN, Gabriel; OLIVEIRA, Matheus Henrique Marques de. A discriminação de mulheres travestis e transexuais no mercado de trabalho. Revista Científica Hermes FIPEN, [S.1.], v. 14, p. 201-218, nov. 2015. ISSN 2175-0556. Disponível em:

<http://www.fipen.edu.br/hermes1/index.php/hermes1/article/view/210>. Acesso em: 28 jun. 2021. DOI: http://dx.doi.org/10.21710/rch.v14i0.210.

NOGUEIRA, Sayonara NB; AQUINO, Tathiane Araújo; CABRAL, Euclides Afonso. Dossiê: a geografia dos corpos das pessoas trans. Aracaju: Rede Trans Brasil, 2017. Disponível em

http://redetransbrasil.org.br/arquivo-do-monitoramento/a-geografia-dos-corpos-trans. Acesso em $17 \mathrm{fev}$. 2021.

OIT. UNAIDS. PNUD. Promoção dos Direitos Humanos de pessoas LGBT no Mundo do Trabalho. Projeto "Construindo a igualdade de oportunidades no mundo do trabalho: combatendo a homo-lesbotransfobia. Brasília, 2015. 2a. ed.

PRODANOV, C Ernani Cesar. ROHM, Ricardo Henry Dias; CABRAL, A.; FERNANDES, F. Diversidade sexual e vantagem competitiva: uma análise de seus impactos nas organizações. 2012.

SALES, Ricardo Gonçalves de. Políticas de respeito à diversidade sexual no ambiente de trabalho: análise das percepções sobre o papel da comunicação em organizações participantes de empresas e direitos LGBT.P.134, São Paulo. 2017. Disponível em: https://prceu.usp.br/wp-

content/uploads/2020/07/RICARDOGONCALVESDESALESVC.pdf. Acesso em: 02 jul. 2021.

STILLWELL, Susan B.; FINEOUT-OVERHOLT, Ellen; MELNYK, Bernadette Mazurek; WILLIAMSON, Kathleen M. Searching for the Evidence: Strategies to help you conduct a successful search. American Journal of Nursing (AJN), jan.2010, v. 110, n.1. p. 51-53. Disponível em: http://download.lww.com/wolterskluwer_vitalstream_com/PermaLink/NCNJ/A/NCNJ_546_156_2010_0 8_23_SADFJO_165_SDC216.pdf. Acesso em: 05 jun. 2014.

SOUZA, Luiz Henrique Braúna Lopes de. TRABALHO E DIVERSIDADE SEXUAL E DE GÊNERO: Dilemas entre a inserção econômica e social no mercado de trabalho e as estratégias de sobrevivência da população LGBT. 2020. V.03, N.10. Disponível em: https://periodicoscientificos.ufmt.br/ojs/index.php/rebeh/article/view/10443. Acesso em: 02 jul. 2021.

WARD, USHER-SMITH, GRIFFIN. How to produce a systematic review. InnovAiT. 2019. [In press].

Recebido em: 01/10/2021

Aprovado em: 25/10/2021

Publicado em: 28/10/2021 